\title{
UNIFORM CONVERGENCE OF THE COLLOCATION METHOD FOR PRANDTL'S INTEGRO-DIFFERENTIAL EQUATION
}

\author{
M. R. CAPOBIANCO ${ }^{1}$, G. CRISCUOLO ${ }^{2}$, P. JUNGHANNS ${ }^{3}$ and U. LUTHER ${ }^{3}$
}

\section{Dedicated to Prof. D. Elliott on the occasion of his 65th birthday}

(Received 16 March 1998)

\begin{abstract}
An integro-differential equation of Prandtl's type and a collocation method as well as a collocation-quadrature method for its approximate solution is studied in weighted spaces of continuous functions.
\end{abstract}

\section{Introduction}

This paper is devoted to the investigation of collocation and discrete collocation (or collocation-quadrature) methods for the approximate solution of singular integrodifferential equations of Prandtl's type

$$
g(x) v(x)+a v^{\prime}(x)+\frac{b}{\pi} \int_{-1}^{1} \frac{v^{\prime}(t)}{t-x} d t+\frac{1}{\pi} \int_{-1}^{1} h(x, t) v(t) d t=f(x),
$$

$-1<x<1$, with the additional conditions,

$$
v(-1)=v(1)=0
$$

where $a, b \in \mathbb{B}$ are given constants and $g, f:[-1,1] \rightarrow \mathbb{C}, h:[-1,1]^{2} \rightarrow \mathbb{C} \times \mathbb{C}$ are given functions. In [3] the authors consider a collocation and a collocationquadrature method with the help of weighted Sobolev spaces (see also Section 2 of the

\footnotetext{
${ }^{1}$ Istituto per Applicazioni della Matematica, C.N.R., Via Pietro Castellino 111, 80131 Napoli, Italy. ${ }^{2}$ Dipartimento di Matematica, Universitá degli Studi Napoli "Frederico II", Edificio T Complesso Monte Sant' Angelo, Via Cinthia, 80126 Napoli, Italy.

${ }^{3}$ Technische Universität Chemnitz, Fakultät für Mathematik, D-09107 Chemnitz, Germany.

(C) Australian Mathematical Society 2000, Serial-fee code 0334-2700/00
} 
present paper) and optimal convergence rates are proved in respective Sobolev norms. Here we investigate a regularized version of (1.1) in a scale of weighted Besov spaces as subspaces of weighted spaces of continuous functions in order to obtain convergence results for the above mentioned approximation methods in uniform norms. Weighted uniform convergence results are obtained, for example, in [2, 4, 5, 8]. Weighted Besov spaces are introduced and used as a powerful tool in studying the uniform convergence of polynomial approximation methods for Cauchy singular integral equations in [6]. The aim of the present paper is to show that this tool works also in the case of singular integro-differential (or hypersingular integral) equations of the form (1.1).

In Section 2 we recall some mapping properties of singular integral operators in weighted Sobolev spaces as well as in weighted spaces of continuous functions and give a representation of the inverse operator of the hypersingular integral operator involved in (1.1). With the help of this representation we prove the boundedness of this operator in a scale of pairs of weighted Besov spaces. In Section 3 we describe the collocation and collocation-quadrature methods under consideration and prove their convergence as well as error estimates in weighted Besov norms. Finally, in Section 4 we make some remarks on a fast algorithm considered in [3], the uniform convergence of which can be investigated with the technique presented in [7].

\section{Mapping properties}

Let $a$ and $b<0$ be real numbers with $a^{2}+b^{2}=1$ and define $\alpha, 0<\alpha<1$, and $\beta=1-\alpha$, by $a-i b=e^{i \pi \alpha}$. By $v^{\alpha, \beta}$ and $A$ we denote the Jacobi weight function $v^{\alpha, \beta}(x)=(1-x)^{\alpha}(1+x)^{\beta}$ and the Cauchy singular integral operator

$$
(A u)(x)=a v^{\alpha, \beta}(x) u(x)+\frac{b}{\pi} \int_{-1}^{1} \frac{u(t)}{t-x} v^{\alpha, \beta}(t) d t, \quad-1<x<1,
$$

respectively. If $D=d / d x$ denotes the operator of generalized differentiation, then the hypersingular integral operator $V=D A$ is given by

$$
(V u)(x)=\frac{d}{d x}\left[a v^{\alpha, \beta}(x) u(x)+\frac{b}{\pi} \int_{-1}^{1} \frac{u(t)}{t-x} v^{\alpha, \beta}(t) d t\right]
$$

(cf. [3, Section 2]). For $\gamma>-1$ and $\delta>-1$, let $\mathbf{L}_{\gamma, \delta}^{2}$ denote the weighted space of complex-valued and square integrable functions on the interval $[-1,1]$ endowed with the inner product and the norm

$$
\langle u, v\rangle_{\gamma, \delta}=\frac{1}{\pi} \int_{-1}^{1} u(x) \overline{v(x)} v^{\gamma, \delta}(x) d x \quad \text { and } \quad\|u\|_{\gamma, \delta}=\sqrt{\langle u, u\rangle_{\gamma, \delta}},
$$


respectively. For real numbers $s \geq 0$ define the weighted Sobolev space $\mathbf{L}_{\gamma, \delta}^{2, s}$ by

$$
\mathbf{L}_{\gamma, \delta}^{2, s}=\left\{u \in \mathbf{L}_{\gamma, \delta}^{2}: \sum_{n=0}^{\infty}(1+n)^{2 s}\left|\left\langle u, p_{n}^{\gamma, \delta}\right\rangle_{\gamma, \delta}\right|^{2}<\infty\right\},
$$

where $p_{n}^{\gamma, \delta}$ is the normalized (with respect to $\langle., .\rangle_{\gamma, \delta}$ ) Jacobi polynomial (with positive leading coefficient) of degree $n$ and the norm in $\mathbf{L}_{\gamma, \delta}^{2, s}$ is given by

$$
\|u\|_{\gamma, \delta, s}=\left(\sum_{n=0}^{\infty}(1+n)^{2 s}\left|\left\langle u, p_{n}^{\gamma, \delta}\right\rangle_{\gamma, \delta}\right|^{2}\right)^{1 / 2}
$$

$\mathbf{L}_{\gamma, \delta}^{2, s}$ is a Banach space for all $s \geq 0$ and compactly embedded into $\mathbf{L}_{\gamma, \delta}^{2, t}$ for $0 \leq t<s$ (cf. [1, Conclusion 2.3]).

PROPOSITION 2.1 ([3, Corollary 2.8]). For each $s \geq 0$, the hypersingular integral operator $V$ defined by (2.2) is a continuous isomorphism between $\mathbf{L}_{\alpha, \beta}^{2, s+1}$ and $\mathbf{L}_{\beta, \alpha}^{2, s}$. Moreover, for $u \in \mathbf{L}_{\alpha, \beta}^{2, s+1}$,

$$
V u=\sum_{n=0}^{\infty}(n+1)\left\langle u, p_{n}^{\alpha, \beta}\right\rangle_{\alpha, \beta} p_{n}^{\beta, \alpha}
$$

where the series converges in the sense of $\mathbf{L}_{\beta, \alpha}^{2, s}$.

Now our first aim is to describe the inverse operator of $V: \mathbf{L}_{\alpha, \beta}^{2, s+1} \rightarrow \mathbf{L}_{\beta, \alpha}^{2, s}$. After this we will study the mapping properties of $V^{-1}$ in appropriate pairs of weighted spaces of continuous functions. In view of Proposition 2.1 , the operator $V^{-1}: \mathbf{L}_{\beta, \alpha}^{2, s} \rightarrow \mathbf{L}_{\alpha, \beta}^{2, s+1}$ is uniquely determined by

$$
V^{-1} p_{n}^{\beta, \alpha}=\frac{1}{n+1} p_{n}^{\alpha, \beta}, \quad n=0,1,2, \ldots,
$$

and by continuity (since the set of polynomials is dense in $\mathbf{L}_{\gamma, \delta}^{2, s}$ ).

Let us remember some mapping properties of Cauchy singular integral operators of type (2.1). If $\mathbf{X}$ and $\mathbf{Y}$ are Banach spaces then by $\mathscr{L}(\mathbf{X}, \mathbf{Y})$ we denote the space of linear and bounded operators from $\mathbf{X}$ into $\mathbf{Y}$. In the case of $\mathbf{X}=\mathbf{Y}$ we write $\mathscr{L}(\mathbf{X})$ instead of $\mathscr{L}(\mathbf{X}, \mathbf{X})$.

LEMMA 2.2 ([13, Theorem 9.14], [1, Lemma 4.1]). If $\lambda$ and $v$ are integers, such that

$$
-1<\gamma:=\lambda+\alpha, \quad \delta:=\nu-\alpha<1,
$$


and

$$
\begin{aligned}
& \left(A_{\gamma, \delta} u\right)(x)=a v^{\gamma, \delta}(x) u(x)+\frac{b}{\pi} \int_{-1}^{1} \frac{u(t)}{t-x} v^{\gamma, \delta}(t) d t, \\
& \left(\widehat{A}_{\gamma, \delta} f\right)(t)=a v^{-\gamma,-\delta}(t) f(t)-\frac{b}{\pi} \int_{-1}^{1} \frac{f(x)}{x-t} v^{-\gamma,-\delta}(x) d x,
\end{aligned}
$$

then $A_{\gamma, \delta} \in \mathscr{L}\left(\mathbf{L}_{\gamma, \delta}^{2, s}, \mathbf{L}_{-\gamma,-\delta}^{2, s}\right)$ and $\widehat{A}_{\gamma, \delta} \in \mathscr{L}\left(\mathbf{L}_{-\gamma,-\delta}^{2, s}, \mathbf{L}_{\gamma, \delta}^{2, s}\right)$ for all $s \geq 0$. Moreover, for $\kappa:=-(\lambda+\nu)=-(\gamma+\delta)$,

$$
A_{\gamma, \delta} p_{n}^{\gamma, \delta}=(-1)^{\lambda} p_{n-\kappa}^{-\gamma,-\delta}, \quad \widehat{A}_{\gamma, \delta} p_{n}^{-\gamma,-\delta}=(-1)^{\lambda} p_{n+\kappa}^{\gamma, \delta}, \quad n=0,1,2, \ldots,
$$

$\left(p_{-1}^{\gamma, \delta}=p_{-1}^{-\gamma,-\delta} \equiv 0\right)$ and $\widehat{A}_{\gamma, \delta} A_{\gamma, \delta}=I$, if $\kappa \leq 0$, as well as $A_{\gamma, \delta} \widehat{A}_{\gamma, \delta}=I$, if $\kappa \geq 0$.

PROPOSITION 2.3. The inverse operator $V^{-1}: \mathbf{L}_{\beta, \alpha}^{2, s} \rightarrow \mathbf{L}_{\alpha, \beta}^{2, s+1}$ of the hypersingular integral operator (2.2) can be written in the form

$$
V^{-1}=\widehat{V}:=\widehat{A} W B
$$

where the continuous operators $B: \mathbf{L}_{\beta, \alpha}^{2, s} \rightarrow \mathbf{L}_{-\beta,-\alpha}^{2, s}, W: \mathbf{L}_{-\beta,-\alpha}^{2, s} \rightarrow \mathbf{L}_{\beta-1, \alpha-1}^{2, s+1}=$ $\mathbf{L}_{-\alpha,-\beta}^{2, s+1}$ and $\widehat{A}: \mathbf{L}_{-\alpha,-\beta}^{2, s+1} \rightarrow \mathbf{L}_{\alpha, \beta}^{2, s+1}$ are defined by

$$
\begin{aligned}
& (B u)(x)=a v^{\beta, \alpha}(x) u(x)-\frac{b}{\pi} \int_{-1}^{1} \frac{u(t)}{t-x} v^{\beta, \alpha}(t) d t \\
& (W u)(x)=a \int_{-1}^{x} v^{-\beta,-\alpha}(t) u(t) d t-\frac{b}{\pi} \int_{-1}^{1} v^{-\beta,-\alpha}(t) \ln |x-t| u(t) d t
\end{aligned}
$$

and

$$
(\widehat{A} u)(x)=a v^{-\alpha,-\beta}(x) u(x)-\frac{b}{\pi} \int_{-1}^{1} \frac{u(t)}{t-x} v^{-\alpha,-\beta}(t) d t,
$$

respectively.

PROOF. If we choose in Lemma $2.2 \gamma=\alpha-1$ and $\delta=-\alpha$, then $\beta=-\gamma, \alpha=-\delta$ and $B=\widehat{A}_{\gamma, \delta}$. Thus

$$
B \in \mathscr{L}\left(\mathbf{L}_{\beta, \alpha}^{2, s}, \mathbf{L}_{-\beta,-\alpha}^{2, s}\right) \quad \text { and } B p_{n}^{\beta, \alpha}=-p_{n+1}^{-\beta,-\alpha}, \quad n=0,1,2, \ldots
$$

From the generalized Rodrigues' formula [14, (4.10.1)] it follows, for arbitrary $\gamma>-1, \delta>-1$ and $n=1,2, \ldots$,

$$
\begin{aligned}
& (1-x)^{\gamma}(1+x)^{\delta} p_{n}^{\gamma, \delta}(x) \\
& \quad=-[n(n+\gamma+\delta+1)]^{-1 / 2} \frac{d}{d x}\left[(1-x)^{\gamma+1}(1+x)^{\delta+1} p_{n-1}^{\gamma+1, \delta+1}(x)\right],
\end{aligned}
$$


which implies (for $\gamma=-\beta, \delta=-\alpha$ )

$$
\int_{-1}^{x} v^{-\beta,-\alpha}(t) p_{n+1}^{-\beta,-\alpha}(t) d t=-\frac{1}{n+1} v^{\alpha, \beta}(x) p_{n}^{\alpha, \beta}(x), \quad n=0,1,2, \ldots,
$$

and, with the help of partial integration,

$$
\int_{-1}^{1} v^{-\beta,-\alpha}(t) \ln |x-t| p_{n+1}^{-\beta,-\alpha}(t) d t=\frac{1}{n+1} \int_{-1}^{1} \frac{p_{n}^{\alpha, \beta}(t)}{t-x} v^{\alpha, \beta}(t) d t, \quad n=0,1,2, \ldots
$$

Consequently, for $n=0,1,2, \ldots$,

$$
W p_{n+1}^{-\beta,-\alpha}=-\frac{1}{n+1} A p_{n}^{\alpha, \beta}=-\frac{1}{n+1} A_{\alpha, \beta} p_{n}^{\alpha, \beta}=-\frac{1}{n+1} p_{n+1}^{-\alpha,-\beta},
$$

taking into account Lemma 2.2. Again by Lemma 2.2, we have

$$
\widehat{A}=\widehat{A}_{\alpha, \beta} \in \mathscr{L}\left(\mathbf{L}_{-\alpha,-\beta}^{2, s+1}, \mathbf{L}_{\alpha, \beta}^{2, s+1}\right)
$$

and

$$
\widehat{A} p_{n+1}^{-\alpha,-\beta}=\widehat{A}_{\alpha, \beta} p_{n+1}^{-\alpha,-\beta}=p_{n}^{\alpha, \beta}, \quad n=0,1,2, \ldots
$$

Putting (2.4), (2.5) and (2.6) together, we obtain

$$
(\widehat{A} W B) p_{n}^{\beta, \alpha}=\frac{1}{n+1} p_{n}^{\alpha, \beta}, \quad n=0,1,2, \ldots .
$$

Thus it remains to prove that $W \in \mathscr{L}\left(\mathbf{L}_{-\beta,-\alpha}^{2, s}, \mathbf{L}_{-\alpha,-\beta}^{2, s+1}\right)$. Obviously $W: \mathbf{L}_{-\beta,-\alpha}^{2} \rightarrow$ $\mathbf{L}_{-\alpha,-\beta}^{2}$ is continuous. Now, let $u \in \mathbf{L}_{-\beta,-\alpha}^{2, s}$. Then $u=\sum_{n=0}^{\infty}\left\langle u, p_{n}^{-\beta,-\alpha}\right\rangle_{-\beta,-\alpha} p_{n}^{-\beta,-\alpha}$ (in the sense of $\mathbf{L}_{-\beta,-\alpha}^{2}$ ) and, in view of (2.5),

$$
W u=\left\langle u, p_{0}^{-\beta,-\alpha}\right\rangle_{-\beta,-\alpha} W p_{0}^{-\beta,-\alpha}-\sum_{n=1}^{\infty} \frac{1}{n}\left\langle u, p_{n}^{-\beta,-\alpha}\right\rangle_{-\beta,-\alpha} p_{n}^{-\alpha,-\beta} .
$$

Since, due to Lemma 2.2,

$$
\left(W p_{0}^{-\beta,-\alpha}\right)^{\prime}(x)=a v^{-\beta,-\alpha}(x) p_{0}^{-\beta,-\alpha}(x)+\frac{b}{\pi} \int_{-1}^{1} \frac{p_{0}^{-\beta,-\alpha}(t)}{t-x} v^{-\beta,-\alpha}(t) d t \equiv 0,
$$

we have $W p_{0}^{-\beta,-\alpha}=c_{-\beta_{-}-\alpha} p_{0}^{-\alpha,-\beta}$, where $c_{-\beta_{-}-\alpha}$ is a constant. It follows that

$$
\begin{aligned}
\|W u\|_{-\alpha,-\beta, s+1}^{2} & =\left|c_{-\beta,-\alpha}\right|^{2}\left|\left\langle u, p_{0}^{-\beta,-\alpha}\right\rangle_{-\beta,-\alpha}\right|^{2}+\sum_{n=1}^{\infty} \frac{(1+n)^{2 s+2}}{n^{2}}\left|\left\langle u, p_{n}^{-\beta,-\alpha}\right\rangle_{-\beta,-\alpha}\right|^{2} \\
& \leq c\|u\|_{-\beta,-\alpha, s}^{2} .
\end{aligned}
$$

The proposition is proved. 
For nonnegative real constants $\rho$ and $\tau$, we denote by $\mathbf{C}_{\rho, \tau}$ the Banach space of all continuous functions $u:(-1,1) \rightarrow \mathbb{C}$, where $v^{\rho, r} u$ belongs to the space $\mathbf{C}=\mathbf{C}[-1,1]$ of all continuous functions over $[-1,1]$. The norm in $\mathbf{C}_{\rho, \tau}$ is defined by

$$
\|u\|_{\infty, \rho, \tau}=\left\|u v^{\rho, \tau}\right\|_{\infty}, \quad \text { where } \quad\|v\|_{\infty}:=\sup \{|v(x)|: x \in[-1,1]\} .
$$

Furthermore, let $\mathbf{C}_{\rho, \tau}^{0}$ be the closed subspace of $\mathbf{C}_{\rho, \tau}$ of functions $u \in \mathbf{C}_{\rho, \tau}$ with

$$
\left(v^{\rho, \tau} u\right)(1)=0 \text { if } \rho>0 \text { and }\left(v^{\rho, \tau} u\right)(-1)=0 \text { if } \tau>0 .
$$

Let $\Pi_{n}$ denote the set of polynomials of degree less than $n\left(\Pi_{0}:=\{0\}\right)$. For a function $u \in \mathbf{C}_{\rho, \tau}$, we denote by $E_{n}^{\rho, \tau}(u)$ the best weighted uniform approximation of $u$ by polynomials belonging to $\Pi_{n}$, that is,

$$
E_{n}^{\rho, \tau}(u)=\inf \left\{\|u-p\|_{\infty, \rho, \tau}: p \in \Pi_{n}\right\} .
$$

For constants $\chi>0$ and $q \geq 0$ we define the weighted Besov space

$$
\mathbf{C}_{\rho, \tau}^{\chi, q}=\left\{u \in \mathbf{C}_{\rho, \tau}:\|u\|_{\rho, \tau, x, q}:=\sup \left\{\frac{E_{n}^{\rho, \tau}(u)(n+1)^{x}}{[1+\log (n+1)]^{q}}: n=0,1,2, \ldots\right\}<\infty\right\} .
$$

$\mathbf{C}_{\rho, \tau}^{x, q}$ is a Banach space and compactly embedded into $\mathbf{C}_{\rho, \tau}^{0}$. Moreover, if

$$
\lim _{n \rightarrow \infty} \frac{\log ^{q_{1}-q_{2}} n}{n^{x_{1}-x_{2}}}=0
$$

then $\mathbf{C}_{\rho, \tau}^{\chi_{1}, q_{1}}$ is compactly embedded into $\mathbf{C}_{\rho, \tau}^{x_{2}, q_{2}}$ and if $\rho \leq \rho^{\prime}, \tau \leq \tau^{\prime}$ then $\mathbf{C}_{\rho, \tau}^{\chi, q} \subset \mathbf{C}_{\rho^{\prime}, \tau^{\prime}}^{x, q}$ continuously (see [6, Section 3]). The Bernstein-type inequalities

$$
\|p\|_{\rho, \tau, \chi, q} \leq n^{\chi}\|p\|_{\infty, \rho, \tau}
$$

and

$$
\|p\|_{\rho, \tau, \chi, q} \leq n^{x-\chi^{\prime}}\|p\|_{\rho, \tau, \chi^{\prime}, q}, \quad 0<\chi^{\prime} \leq \chi,
$$

for all $p \in \Pi_{n}$ can be easily checked.

LeMMA 2.4 ([6, Proposition 4.7, Remark 4.9, Remark 4.16]). Let $\gamma$ and $\delta$ be defined as in Lemma 2.2, where $\gamma^{ \pm}$and $\delta^{ \pm}$are nonnegative constants satisfying $\gamma=\gamma^{+}-\gamma^{-}$and $\delta=\delta^{+}-\delta^{-}$. Then

$$
A_{\gamma, \delta} \in \mathscr{L}\left(\mathbf{C}_{\gamma^{+}, \delta^{+}}^{x, q}, \mathbf{C}_{\gamma^{-}, \delta^{-}}^{x,+1}\right) \text { if } \gamma^{-}<1 \text { and } \delta^{-}<1
$$

and

$$
\widehat{A_{\gamma, \delta}} \in \mathscr{L}\left(\mathbf{C}_{\gamma^{-}, \delta^{-}}^{\chi, q}, \mathbf{C}_{\gamma^{+}, \delta^{+}}^{x, q+1}\right) \text { if } \gamma^{+}<1 \text { and } \delta^{+}<1
$$


In all that follows, we denote by $c$ a positive constant which can have different values in different places. Moreover, by $c \neq c(n, f, \ldots)$ we will indicate that $c$ is independent of $n, f, \ldots$ To prove a "sharp" mapping property of $\widehat{V}$ in a scale of pairs of weighted Besov spaces using the representation (2.3) we need the following lemma. Let $\gamma$ and $\delta$ be defined as in Lemma 2.2 and let $\gamma^{ \pm}$and $\delta^{ \pm}$be nonnegative constants satisfying $\gamma^{+}-\gamma^{-}=\gamma, \delta^{+}-\delta^{-}=\delta$ and $\gamma^{ \pm}<1, \delta^{ \pm}<1$. Note that $u \in \mathbf{C}_{\gamma^{-}, \delta^{-}}$ does not in general imply $u \in \mathbf{L}_{\gamma, \delta}^{2}$.

LEMMA 2.5 (cf. [9, Proposition 5.2]). If $\kappa=-(\gamma+\delta) \geq 0$ then $A_{\gamma, \delta} \widehat{A}_{\gamma, \delta} u=u$ for all $u \in \mathbf{C}_{\gamma^{-}, \delta^{-}}^{\chi, q}$

PROOF. Let $u \in \mathrm{C}_{\gamma^{-,}, \delta^{-}}^{X, q}$ and $P_{n} \in \Pi_{n}$ with $\left\|u-P_{n}\right\|_{\infty, \gamma^{-}, \delta^{-}}=E_{n}^{\gamma^{-}, \delta^{-}}(u)$. Then, for $m<n$,

$$
\begin{aligned}
E_{m}^{\gamma^{-}, \delta^{-}}\left(u-P_{n}\right) & \leq\left\|u-P_{n}\right\|_{\infty, \gamma^{-}, \delta^{-}} \\
& \leq c \frac{[1+\log (n+1)]^{q}}{(n+1)^{x}} \leq \frac{c}{(m+1)^{x / 2}} \frac{[1+\log (n+1)]^{q}}{(n+1)^{x / 2}}
\end{aligned}
$$

and, for $m \geq n$,

$$
\begin{aligned}
E_{m}^{\gamma^{-}, \delta^{-}}\left(u-P_{n}\right)=E_{m}^{\gamma^{-}, \delta^{-}}(u) & \leq c \frac{[1+\log (m+1)]^{q}}{(m+1)^{x}} \\
& \leq c \frac{[1+\log (m+1)]^{q}}{(m+1)^{x / 2}} \frac{1}{(n+1)^{x / 2}}
\end{aligned}
$$

Thus

$$
\left\|u-P_{n}\right\|_{\gamma^{-}, \delta^{-}, x / 2, q} \leq c \frac{[1+\log (n+1)]^{q}}{(n+1)^{x / 2}} \longrightarrow 0
$$

From Lemma 2.4 it follows that

$$
\lim _{n \rightarrow \infty}\left\|A_{\gamma, \delta} \widehat{A}_{\gamma, \delta}\left(u-P_{n}\right)\right\|_{\gamma^{-}, \delta^{-}, x / 2, q+2}=0 .
$$

Since, in view of Lemma $2.2, A_{\gamma, \delta} \widehat{A}_{\gamma, \delta} P_{n}=P_{n}$ and $\lim _{n \rightarrow \infty}\left\|u-P_{n}\right\|_{\infty, \gamma^{-}, \delta^{-}}=0$, we obtain the relation $A_{\gamma, \delta} \widehat{A}_{\gamma, \delta} u=u$.

PROPOSITION 2.6. For arbitrary $\chi>0$ and $q \geq 0$, the operator $\widehat{V}$ defined in (2.3) is continuous from $\mathbf{C}_{\gamma^{-}, \delta^{-}}^{x, q}$ into $\mathbf{C}_{\gamma^{+}+1 / 2, \delta^{+}+1 / 2}^{x+1, q+1}$, where $\gamma^{-}-\gamma^{+}=\beta, \delta^{-}-\delta^{+}=\alpha$, $0 \leq \gamma^{+}, \delta^{+}<1 / 2$ and $1 / 2 \leq \gamma^{-}, \delta^{-}<1$. 
Proof. We use the representation $\widehat{V}=\widehat{A} W B$ (see Proposition 2.3). Since $B=$ $\widehat{A_{\gamma, \delta}}$ with $\gamma=\alpha-1=-\beta$ and $\delta=-\alpha$, from Lemma 2.4 it follows that

$$
B \in \mathscr{L}\left(\mathbf{C}_{\gamma^{-}, \delta^{-}}^{x, q}, \mathbf{C}_{\gamma^{+}, \delta^{+}}^{x, q+1}\right)
$$

Now, let $u \in \mathbf{C}_{\gamma^{-,}, \delta^{-}}^{x . q}$ Then $\tilde{u}=B u \in \mathbf{C}_{\gamma^{+}, \delta^{+}}^{x, q+1}$ and

$$
\begin{aligned}
(W B u)^{\prime}(x) & =a v^{-\beta,-\alpha}(x) \widetilde{u}(x)+\frac{b}{\pi} \int_{-1}^{1} \frac{\tilde{u}(t)}{t-x} v^{-\beta,-\alpha}(t) d t \\
& =\left(A_{-\beta,-\alpha} \widetilde{u}\right)(x)=\left(A_{\gamma, \delta} \widehat{A}_{\gamma, \delta} u\right)(x)=u(x),
\end{aligned}
$$

using Lemma 2.5 and $\gamma+\delta=-1$. Taking into account the relation

$$
E_{n}^{\rho, \tau}(f) \leq \frac{c}{n} E_{n-1}^{\rho+1 / 2, \tau+1 / 2}\left(f^{\prime}\right), \quad n=1,2, \ldots
$$

(see, for example, [6, Corollary 3.10], where the assertion is formulated for $n=$ $2,3, \ldots$, since $E_{n}^{\rho, \tau}$ is defined slightly differently from the definition given here), where $f^{\prime} \in \mathbf{C}_{\rho+1 / 2, \tau+1 / 2}$ and $c \neq c(n, f)$, we obtain

$$
E_{n}^{\gamma^{--1 / 2, \delta^{-}-1 / 2}}(W B u) \leq \frac{c}{n} E_{n-1^{-}}^{\gamma^{-} \delta^{-}}(u) \leq c \frac{\log ^{q}(n+1)}{n^{x+1}}\|u\|_{\gamma^{-}, \delta^{-}, x, q}, \quad n=1,2, \ldots
$$

Since, in view of (2.9),

$$
\begin{aligned}
E_{0}^{\gamma^{-}-1 / 2, \delta^{-}-1 / 2}(W B u) & =\|W B u\|_{\infty, \gamma^{-}-1 / 2, \delta^{-}-1 / 2} \\
& \leq c\|W B u\|_{\infty} \leq c\|B u\|_{\infty, \gamma^{+}, \delta^{+}} \leq c\|u\|_{\gamma^{-}, \delta^{-}, \chi, q},
\end{aligned}
$$

we get

$$
W B \in \mathscr{L}\left(\mathbf{C}_{\gamma^{-}, \delta^{-}}^{\chi,}, \mathbf{C}_{\gamma^{-}-1 / 2, \delta^{-}-1 / 2}^{x+1, q}\right) .
$$

To consider $\widehat{A}=\widehat{A}_{\alpha, \beta}$, we set $\alpha^{+}=\gamma^{+}+1 / 2, \beta^{+}=\delta^{+}+1 / 2, \alpha^{-}=\gamma^{-}-1 / 2$, $\beta^{-}=\delta^{-}-1 / 2$ and obtain, with the help of Lemma 2.4 ,

$$
\widehat{A} \in \mathscr{L}\left(\mathbf{C}_{\gamma^{-}-1 / 2, \delta^{-}-1 / 2}^{\chi,}, \mathbf{C}_{\gamma^{+}+1 / 2, \delta^{+}+1 / 2}^{\chi, q+1}\right) .
$$

The assertion now follows from (2.10) and (2.11).

COROLLARY 2.7. For the case $a=0, b=-1$, that is, $\alpha=\beta=1 / 2$, the previous proposition gives $\widehat{V} \in \mathscr{L}\left(\mathbf{C}_{1 / 2,1 / 2}^{x, q}, \mathbf{C}_{1 / 2,1 / 2}^{x+1, q+1}\right)$.

LEMMA 2.8 ([6, Lemma 4.14]). If $f \in \mathbf{C}_{0,0}^{\chi, q}$ then, for all $u \in \mathbf{C}_{\rho, \tau}^{x, q}$,

$$
\|f u\|_{x, q, \rho, \tau} \leq c\|u\|_{x, q, \rho, \tau}
$$

where $c \neq c(u)$, that is, the operator of multiplication by $f$ belongs to $\mathscr{L}\left(\mathbf{C}_{\rho, \tau}^{\chi, q}\right)$. 
In the following lemma we formulate a result on the mapping properties of the operator $K$ given by

$$
(K u)(x)=\frac{1}{\pi} \int_{-1}^{1} h(x, t) u(t) v^{\alpha, \beta}(t) d t, \quad-1<x<1 .
$$

For this, $h \in \mathbf{C}_{\rho, \tau, x}^{x, q} \cap \mathbf{C}_{v, \zeta, t}$ means that the function $G(x, t):=h(x, t) v^{\rho, \tau}(x) v^{v, \zeta}(t)$ is continuous for $(x, t) \in[-1,1]^{2}$ and $h_{t}^{\nu, \zeta}:=h(., t) v^{\nu, \zeta}(t)$ belongs to $\mathbf{C}_{\rho, \tau}^{x, q}$ uniformly with respect to $t \in[-1,1]$.

LEMMA 2.9 ([6, Proposition 4.12]). Let $\alpha^{ \pm}, \beta^{ \pm}, v$ and $\zeta$ be nonnegative numbers satisfying $\alpha=\alpha^{+}-\alpha^{-}, \beta=\beta^{+}-\beta^{-}, \nu+\alpha^{-}<1$ and $\zeta+\beta^{-}<1$. If $\in \mathbf{C}_{\rho, \tau, x}^{X, q} \cap \mathbf{C}_{\nu, \zeta, t}$ then $K \in \mathscr{L}\left(\mathbf{C}_{\alpha^{+}, \beta^{+}}, \mathbf{C}_{\rho, \tau}^{\chi, q}\right)$.

CoRollary 2.10. If $\gamma^{ \pm}$and $\delta^{ \pm}$are defined as in Proposition 2.6, $v+\gamma^{-}<3 / 2$, $\zeta+\delta^{-}<3 / 2$ and $h \in \mathbf{C}_{\rho, \tau, x}^{x, q} \cap \mathbf{C}_{v, \zeta, t}$, then $K \in \mathscr{L}\left(\mathbf{C}_{\gamma^{+}+1 / 2, \delta^{+}+1 / 2}, \mathbf{C}_{\rho, \tau}^{x, q}\right)$.

Proof. One has only to take into account the relations $\alpha=1-\beta=\gamma^{+}+1 / 2-$ $\left(\gamma^{-}-1 / 2\right)$ and $\beta=1-\alpha=\delta^{+}+1 / 2-\left(\delta^{-}-1 / 2\right)$.

\section{The collocation method}

For all that follows we assume that $\gamma^{ \pm}$and $\delta^{ \pm}$are real numbers satisfying

$$
\gamma^{-}-\gamma^{+}=\beta, \quad \delta^{-}-\delta^{+}=\alpha, \quad 0 \leq \gamma^{+}, \delta^{+}<1 / 2, \quad 1 / 2 \leq \gamma^{-}, \delta^{-}<1
$$

(cf. Proposition 2.6) and set $\alpha^{+}=\gamma^{+}+1 / 2, \alpha^{-}=\gamma^{-}-1 / 2, \beta^{+}=\delta^{+}+1 / 2$ and $\beta^{-}=\delta^{-}-1 / 2$.

Let $\left\{X_{n}\right\}_{n=1}^{\infty}$ be a sequence of partitions of the interval $[-1,1], X_{n}=\left\{x_{n 1}, \ldots, x_{n n}\right\}$, with $-1 \leq x_{n n}<x_{n, n-1}<\cdots<x_{n 1} \leq 1$ and $x_{n 1} \neq 1$ if $\rho>0, x_{n n} \neq 1$ if $\tau>0$. We denote the Lagrange interpolation operator with respect to $X_{n}$ by $L_{n}=L^{X_{n}}$, that is, $L_{n} f \in \Pi_{n}$ and $\left(L_{n} f\right)\left(x_{n j}\right)=f\left(x_{n j}\right), j=1, \ldots, n$. The weighted Lebesgue constant $\left\|L_{n}\right\|_{\rho, \tau}$ is defined by

$$
\left\|L_{n}\right\|_{\rho, \tau}:=\sup \left\{\left\|L_{n} f\right\|_{\infty, \rho, \tau}: f \in \mathbf{C}_{\rho, \tau},\|f\|_{\infty, \rho, \tau}=1\right\} .
$$

In [4, Theorem 4.1], [10, Remark 3.3] and [11, Theorems 2.2, 2.3], the authors construct partitions $\left\{X_{n}\right\}_{n=1}^{\infty}$ with corresponding $\left\|L_{n}\right\|_{\rho, \tau}=O(\log n)$, using systems of zeros of orthogonal polynomials with additional nodes. Recalling Proposition 2.3 and assuming that $K \in \mathscr{L}\left(\mathbf{L}_{\alpha, \beta}^{2,1}, \mathbf{L}_{\beta, \alpha}^{2}\right)$ and $f \in \mathbf{L}_{\beta, \alpha}^{2}$, we can write (1.1) for $v=v^{\alpha, \beta} u$, $u \in \mathbf{L}_{\alpha, \beta}^{2,1}$, in the equivalent form

$$
[I+\widehat{V}(\Gamma+K)] u=\widehat{V} f,
$$


where $\Gamma$ denotes the multiplication operator with $\Gamma(x):=g(x) v^{\alpha, \beta}(x)$, which is assumed to be continuous from $\mathbf{L}_{\alpha, \beta}^{2,1}$ to $\mathbf{L}_{\beta, \alpha}^{2}$.

Let $t_{n k}$ and $\omega_{n k}, k=1, \ldots, n$, be the nodes and weights of the Gaussian rule with respect to the weight $v^{\alpha, \beta}(t)$ and define, for $u \in \mathbf{C}_{\alpha^{+}, \beta^{+}}$,

$$
\left(K_{n} u\right)(x)=\sum_{k=1}^{n} \omega_{n k} h\left(x, t_{n k}\right) u\left(t_{n k}\right)
$$

To find an approximate solution of (3.1) we look for a polynomial $u_{n} \in \Pi_{n}$ satisfying

$$
\Gamma\left(x_{n j}\right) u_{n}\left(x_{n j}\right)+\left((V+K) u_{n}\right)\left(x_{n j}\right)=f\left(x_{n j}\right), \quad j=1, \ldots, n,
$$

or

$$
\Gamma\left(x_{n j}\right) u_{n}\left(x_{n j}\right)+\left(\left(V+K_{n}\right) u_{n}\right)\left(x_{n j}\right)=f\left(x_{n j}\right), \quad j=1, \ldots, n .
$$

Since, in view of Proposition 2.1, $\widehat{V}\left(\Pi_{n}\right)=\Pi_{n}$, the last two systems of equations are equivalent to searching for a solution $u_{n} \in \Pi_{n}$ (or $u_{n} \in \mathbf{L}_{\alpha, \beta}^{2, s+1}$ ) of

$$
\left[I+\widehat{V} L_{n}(\Gamma+K)\right] u_{n}=\widehat{V} L_{n} f
$$

or

$$
\left[I+\widehat{V} L_{n}\left(\Gamma+K_{n}\right)\right] u_{n}=\widehat{V} L_{n} f
$$

respectively. In the following we will study (3.1) as well as (3.3) and (3.4) in appropriate pairs of weighted Besov spaces defined above. For this end we need some preliminary results.

LEMMA 3.1 ([7, Lemma 2.1]). If $u \in \mathbf{C}_{\rho, \tau}, u_{n} \in \Pi_{n}$ and

$$
\left\|u-u_{n}\right\|_{\infty, \rho, \tau} \leq c_{0}(u) \frac{\log ^{q}(n+1)}{n^{x}}, \quad n=1,2, \ldots,
$$

then, for $0<\chi^{\prime} \leq \chi$,

$$
\left\|u-u_{n}\right\|_{\rho, \tau, X^{\prime}, q} \leq \frac{2^{x}}{\log ^{q} 2} c_{0}(u) \frac{\log ^{q}(n+1)}{n^{x-X^{\prime}}}, \quad n=1,2, \ldots .
$$

COROLlaRY 3.2. If $f \in \mathbf{C}_{\rho, \tau}^{x, q}$ and $P_{n} \in \Pi_{n}$ with $\left\|P_{n}-f\right\|_{\infty, \rho, \tau}=E_{n}^{\rho, \tau}(f)$, then

$$
\left\|P_{n}-f\right\|_{\rho, \tau, \chi^{\prime}, q} \leq c\|f\|_{\rho, \tau, \chi, q} \frac{\log ^{q}(n+1)}{n^{x-\chi^{\prime}}}, \quad 0<\chi^{\prime} \leq \chi,
$$

where $c \neq c\left(n, \chi^{\prime}, f\right)$. 
COROLlaRY 3.3. If $f \in \mathbf{C}_{\rho, \tau}^{\chi, q}$ and $\rho \leq \gamma^{-}, \tau \leq \delta^{-}$, then, for $0<\chi^{\prime} \leq \chi$,

$$
\left\|\widehat{V}\left(f-L_{n} f\right)\right\|_{\alpha^{+}, \beta^{+}, \chi^{\prime}+1, q+1} \leq c \frac{\log ^{q}(n+1)}{n^{x-\chi^{\prime}}}\left\|L_{n}\right\|_{\rho, \tau}\|f\|_{\rho, \tau, x, q},
$$

where $c \neq c\left(n, \chi^{\prime}, f\right)$.

Proof. Let $f \in \mathrm{C}_{\rho, \tau}^{x, q}$ and $P_{n} \in \Pi_{n}$ with $\left\|f-P_{n}\right\|_{\infty, \rho, \tau}=E_{n}^{\rho, \tau}(f)$. Then, by the definition of the space $\mathbf{C}_{\rho, \tau}^{X, q}$,

$$
\left\|f-P_{n}\right\|_{\infty, \rho, \tau} \leq c \frac{\log ^{q}(n+1)}{n^{x}}\|f\|_{\rho, \tau, \chi, q} .
$$

Consequently, in view of Corollary 3.2 and (2.7),

$$
\begin{aligned}
\left\|f-L_{n} f\right\|_{\rho, \tau, x^{\prime}, q} & \leq\left\|f-P_{n}\right\|_{\rho, \tau, \chi^{\prime}, q}+\left\|L_{n}\left(f-P_{n}\right)\right\|_{\rho, \tau, \chi^{\prime}, q} \\
& \leq c \frac{\log ^{q}(n+1)}{n^{x-\chi^{\prime}}}\|f\|_{\rho, \tau, \chi, q}+n^{x^{\prime}}\left\|L_{n}\right\|_{\rho, \tau}\left\|f-P_{n}\right\|_{\infty, \rho, \tau} \\
& \leq c \frac{\log ^{q}(n+1)}{n^{x-\chi^{\prime}}}\left\|L_{n}\right\|_{\rho, \tau}\|f\|_{\rho, \tau, \chi, q},
\end{aligned}
$$

which implies, together with Proposition 2.6, the assertion.

COROLlARY 3.4. Let $a=0, b=-1, \gamma^{-}=\delta^{-}=1 / 2, \gamma^{+}=\delta^{+}=0, \chi>0$, $\varepsilon>0$ and $0<\varepsilon^{\prime} \leq \chi$. If $\Gamma \in \mathbf{C}_{0,0}^{x+\varepsilon, q}$ and

$$
\lim _{n \rightarrow \infty} \frac{\log ^{q}(n+1)}{n^{\varepsilon^{\prime}}}\left\|L_{n}\right\|_{1 / 2,1 / 2}=0,
$$

then

$$
\left\|\widehat{V}\left(\Gamma u-L_{n} \Gamma u\right)\right\|_{1 / 2,1 / 2, x^{\prime}+1, q+1} \leq c \frac{\log ^{q}(n+1)}{n^{\tilde{x}-x^{\prime}}}\left\|L_{n}\right\|_{1 / 2,1 / 2}\|u\|_{1 / 2,1 / 2, \tilde{x}, q}
$$

for all $u \in \mathrm{C}_{1 / 2,1 / 2}^{\tilde{\chi} \cdot q}$ and $0<\chi^{\prime} \leq \tilde{\chi} \leq \chi+\varepsilon$, where $c \neq c\left(n, \chi^{\prime}, u\right)$, and

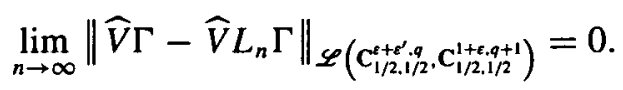

ProOF. Let $u \in \mathbf{C}_{1 / 2,1 / 2}^{\tilde{x}, q}$. Then, by Lemma $2.8 \Gamma u \in \mathbf{C}_{1 / 2,1 / 2}^{\tilde{x}, q}$, and (3.5) follows from Corollary 3.3. Choosing $\chi^{\prime}=\varepsilon$ and $\tilde{\chi}=\varepsilon+\varepsilon^{\prime}$ leads to (3.6).

COROLlaRY 3.5. If $h \in \mathbf{C}_{\rho, \tau, x}^{x+\varepsilon, q} \cap \mathbf{C}_{\nu, \zeta, t}$ for some $\chi>0, \varepsilon>0, v+\gamma^{-}<3 / 2$, $\zeta+\delta^{-}<3 / 2$ and $\rho \leq \gamma^{-}, \tau \leq \delta^{-}$, and if

$$
\lim _{n \rightarrow \infty} \frac{\log ^{q}(n+1)}{n^{x}}\left\|L_{n}\right\|_{\rho, \tau}=0,
$$


then

$$
\left\|\widehat{V}\left(K u-L_{n} K u\right)\right\|_{\alpha^{+}, \beta^{+}, x^{\prime}+1, q+1} \leq c \frac{\log ^{q}(n+1)}{n^{x+\varepsilon-\chi^{\prime}}}\left\|L_{n}\right\|_{\rho, \tau}\|u\|_{\infty, \alpha^{+}, \beta^{+}}
$$

for all $u \in \mathbf{C}_{\alpha^{+}, \beta^{+}}$and $0<\chi^{\prime} \leq \chi+\varepsilon$, where $c \neq c\left(n, \chi^{\prime}, u\right)$, and

$$
\lim _{n \rightarrow \infty}\left\|\widehat{V} K-\widehat{V} L_{n} K\right\|_{\mathscr{L}\left(\mathbf{c}_{\alpha^{+}, \beta^{+}}, \mathbf{c}_{\alpha^{+}, \beta^{+}}^{++1.9+1}\right)}=0
$$

Proof. Let $u \in \mathbf{C}_{\alpha^{+}, \beta^{+}}$. Then, by Corollary 2.10, $K u \in \mathbf{C}_{\rho, \tau}^{\chi+\varepsilon, q}$ and (3.7) follows from Corollary 3.3. The second assertion we get from the first one for $\chi^{\prime}=\varepsilon$.

Now we are able to prove the main results of the present paper. For all that follows we assume that $0 \leq \rho \leq \gamma^{-}$and $0 \leq \tau \leq \delta^{-}$.

THEOREM 3.6. Consider the case $\Gamma \equiv 0$ and assume that

(a) $h \in \mathbf{C}_{\rho, \tau, x}^{x+\varepsilon, q} \cap \mathbf{C}_{\nu, \zeta, t}$ for some $\chi>0, \varepsilon>0, v+\gamma^{-}<3 / 2$ and $\zeta+\delta^{-}<3 / 2$,

(b) $\lim _{n \rightarrow \infty} \frac{\log ^{9}(n+1)}{n^{x}}\left\|L_{n}\right\|_{\rho, \tau}=0$,

and that the homogeneous equation (3.1) possesses only the trivial solution in $\mathrm{C}_{\alpha^{+}, \beta^{+}}^{\mathcal{+}+1, q}$. Then, for each $f \in \mathbf{C}_{\boldsymbol{\rho}, \tau}^{x+\varepsilon, q}$, (3.1) has a unique solution $u^{*} \in \mathbf{C}_{\alpha^{+}, \beta^{+}}^{x+\varepsilon+1, q+1}$. Moreover, for all sufficiently large $n$, the collocation equations (3.3) possess a unique solution $u_{n}^{*}$ and

$$
\left\|u^{*}-u_{n}^{*}\right\|_{\alpha^{+}, \beta^{+}, \chi^{\prime}+1, q+1} \leq c \frac{\log ^{q}(n+1)}{n^{x+\varepsilon-\chi^{\prime}}}\left\|L_{n}\right\|_{\rho, \tau}\|f\|_{\rho, \tau, \chi+\varepsilon, q},
$$

$\varepsilon \leq \chi^{\prime} \leq \chi+\varepsilon$, where $c \neq c\left(n, \chi^{\prime}, f\right)$.

Proof. In view of Lemma 2.9 and Proposition 2.6, the operator $\widehat{V} K: \mathbf{C}_{\alpha^{+}, \beta^{+}}^{x^{\prime}+1, q+1} \longrightarrow$ $\mathbf{C}_{\alpha^{+}, \beta^{+}}^{\chi^{\prime}+1 .+1}$ is compact for $0<\chi^{\prime} \leq \chi+\varepsilon$. Thus $(I+\widehat{V} K)^{-1} \in \mathscr{L}\left(\mathbf{C}_{\alpha^{+}, \beta^{+}}^{\chi^{\prime}+1, q+1}\right)$ exists, and, since $\widehat{V} f \in \mathbf{C}_{\alpha^{+}, \beta^{+}}^{x+\varepsilon+1, q+1}$, (3.1) has a unique solution $u^{*} \in \mathbf{C}_{\alpha^{+}, \beta^{+}}^{x+\varepsilon+, q+1}$. From (3.8) it follows that the inverses $\left(I+\widehat{V} L_{n} K\right)^{-1}: \mathbf{C}_{\alpha^{+}, \beta^{+}}^{\varepsilon+1, q+1} \rightarrow \mathbf{C}_{\alpha^{+}, \beta^{+}}^{\varepsilon+1, q+1}$ exist for all sufficiently large $n$ and are uniformly bounded. Using (2.8) we get, for $P_{n} \in \Pi_{n}$ with $\left\|P_{n}-u^{*}\right\|_{\infty, \alpha^{+}, \beta^{+}}=E_{n}^{\alpha^{+}, \beta^{+}}\left(u^{*}\right)$ and $\varepsilon \leq \chi^{\prime} \leq \chi+\varepsilon$,

$$
\begin{aligned}
\| P_{n} & -u_{n}^{*} \|_{\alpha^{+}, \beta^{+}, x^{\prime}+1, q+1} \\
& \leq n^{x^{\prime}-\varepsilon}\left(\left\|P_{n}-u^{*}\right\|_{\alpha^{+}, \beta^{+}, \varepsilon+1, q+1}+\left\|u^{*}-u_{n}^{*}\right\|_{\alpha^{+}, \beta^{+}, \varepsilon+1, q+1}\right) .
\end{aligned}
$$

By Corollary 3.2 we have

$$
\left\|P_{n}-u^{*}\right\|_{\alpha^{+}, \beta^{+}, \varepsilon+1, q+1} \leq c \frac{\log ^{q+1}(n+1)}{n^{x}}\left\|u^{*}\right\|_{\alpha^{+}, \beta^{+}, x+\varepsilon+1, q+1} .
$$


Furthermore,

$$
\begin{aligned}
\| u^{*} & -u_{n}^{*} \|_{\alpha^{+}, \beta^{+}, \varepsilon+1, q+1} \\
& \leq c\left\|\left(I+\widehat{V} L_{n} K\right) u^{*}-\widehat{V} L_{n} f\right\|_{\alpha^{+}, \beta^{+}, \varepsilon+1, q+1} \\
& \leq c\left(\left\|\widehat{V}\left(f-L_{n} f\right)\right\|_{\alpha^{+}, \beta^{+}, \varepsilon+1, q+1}+\left\|\widehat{V}\left(L_{n} K-K\right) u^{*}\right\|_{\alpha^{+}, \beta^{+}, \varepsilon+1, q+1}\right) \\
& \leq c \frac{\log ^{q}(n+1)}{n^{x}}\left\|L_{n}\right\|_{\rho, \tau}\left(\|f\|_{\rho, \tau, x+\varepsilon, q}+\left\|u^{*}\right\|_{\infty, \alpha^{+}, \beta^{+}}\right),
\end{aligned}
$$

taking into account Corollary 3.3 and (3.7). Together with (3.10) and (3.11) we get

$$
\left\|u^{*}-u_{n}^{*}\right\|_{\alpha^{+}, \beta^{+}, \chi^{\prime}+1, q+1} \leq c \frac{\log ^{q}(n+1)}{n^{x+\varepsilon-\chi^{\prime}}}\left[\left\|L_{n}\right\|_{\rho, \tau}+\log (n+1)\right]\|f\|_{\rho, \tau, x+\varepsilon, q} .
$$

Since

$$
\left\|L_{n}\right\|_{\rho, \tau} \geq c \log (n+1)
$$

(see $[15,16])$, the error estimate $(3.9)$ is proved.

THEOREM 3.7. Assume $a=0, b=-1$ (that is, $\alpha=\beta=1 / 2$ ) and that

(a) $\Gamma \in \mathbf{C}_{0,0}^{\chi+\varepsilon, q}$ for some $\chi>0$ and $\varepsilon>0$,

(b) $h \in \mathbf{C}_{1 / 2,1 / 2, x}^{x+\varepsilon,} \cap \mathbf{C}_{\nu, \zeta, t}$ for some $v<1$ and $\zeta<1$,

(c) $\lim _{n \rightarrow \infty} \frac{\log ^{q}(n+1)}{n^{\varepsilon^{\prime}}}\left\|L_{n}\right\|_{1 / 2,1 / 2}=0$ for some $0<\varepsilon^{\prime}<\min \{1, \chi\}$,

and that the homogeneous equation (3.1) possesses only the trivial solution in $\mathbf{C}_{1 / 2,1 / 2}^{\varepsilon+1, q+1}$. Then, for each $f \in \mathbf{C}_{1 / 2,1 / 2}^{x+\varepsilon, q}$, (3.1) has a unique solution $\mathbf{u}^{*} \in \mathbf{C}_{1 / 2,1 / 2}^{x+\varepsilon+1, q+1}$. Moreover, for all sufficiently large $n,(3.3)$ possesses a unique solution $u_{n}^{*}$ and, for $\varepsilon \leq \chi^{\prime} \leq \chi+\varepsilon$,

$$
\left\|u^{*}-u_{n}^{*}\right\|_{1 / 2,1 / 2, x^{\prime}+1, q+1} \leq c \frac{\log ^{q}(n+1)}{n^{x+\varepsilon-x^{\prime}}}\left\|L_{n}\right\|_{1 / 2,1 / 2}\|f\|_{1 / 2,1 / 2, x+\varepsilon, q},
$$

where $c \neq c\left(n, \chi^{\prime}, f\right)$.

PROOF. By Lemma 2.8 and Corollary 2.7, the operator $\widehat{V} \Gamma: \mathbf{C}_{1 / 2,1 / 2}^{x^{\prime}+1, q+1} \rightarrow$ $\mathbf{C}_{1 / 2,1 / 2}^{x^{\prime}+1, q+1}$ is compact for $0<\chi^{\prime} \leq \chi+\varepsilon$. The compactness of $\widehat{V} K: \mathbf{C}_{1 / 2,1 / 2}^{x^{\prime}+1, q+1} \rightarrow$ $\mathbf{C}_{1 / 2,1 / 2}^{x^{\prime}+1, q+1}$ as well as the unique existence of the solution $u^{*} \in \mathbf{C}_{1 / 2,1 / 2}^{x+\varepsilon+1, q+1}$ of (3.1) are proved as in the proof of Theorem 3.6. From (3.6) and (3.8) it follows that the inverses $\left[I+\widehat{V} L_{n}(\Gamma+K)\right]^{-1}: \mathbf{C}_{1 / 2,1 / 2}^{\varepsilon+1, q+1} \longrightarrow \mathbf{C}_{1 / 2,1 / 2}^{\varepsilon+1, q+1}$ exist for all sufficiently large $n$ and are uniformly bounded. In the same way as in the proof of Theorem 3.6 we obtain the estimation (3.13) using relation (3.5) for $\chi^{\prime}=\varepsilon$ and $\tilde{\chi}=\chi+\varepsilon$.

To investigate the collocation-quadrature method (3.4) we need the following lemma, the proof of which can be found in [6, proof of Theorem 6.8]. 
LEMMA 3.8. If $h \in \mathbf{C}_{\rho, \tau, x}^{x, q} \cap \mathbf{C}_{v, \zeta, t}^{\psi, l}$, that is, $h \in \mathbf{C}_{\rho, \tau, x}^{x, q} \cap \mathbf{C}_{v, \zeta, t}$ and $h(x,.) v^{\rho, \tau}(x) \in$ $\mathbf{C}_{\nu, \zeta}^{\psi, l}$ uniformly with respect to $x \in[-1,1]$, where $\nu+\gamma^{-}<3 / 2$ and $\zeta+\delta^{-}<3 / 2$, then

$$
\left\|\left(K_{n}-K\right) p\right\|_{\infty, \rho, \tau} \leq c \frac{\log ^{l}(n+1)}{n^{\psi}}\|p\|_{\infty, \alpha^{+}, \beta^{+}}
$$

for all $p \in \Pi_{n}$, where $K_{n}$ is defined in (3.2) and $c \neq c(n, p)$.

THEOREM 3.9. Let $\Gamma \equiv 0$ and assume that

(a) $h \in \mathbf{C}_{\rho, \tau, x}^{x+\varepsilon, q} \cap \mathbf{C}_{v, \zeta, q}^{x+\varepsilon, q}$ for some $\chi>0, \varepsilon>0, \nu+\gamma^{-}<3 / 2$ and $\zeta+\delta^{-}<3 / 2$, (b) $\lim _{n \rightarrow \infty} \frac{\log ^{q}(n+1)}{n^{x}}\left\|L_{n}\right\|_{\rho, \tau}=0$,

and that the homogeneous equation (3.1) has only the trivial solution in $\mathbf{C}_{\alpha^{+}, \beta^{+}}^{\varepsilon+1}$. Then, for each $f \in \mathbf{C}_{\rho, \tau}^{x+\varepsilon, q}$, (3.1) has a unique solution $u^{*} \in \mathbf{C}_{\alpha^{+}, \beta^{+}}^{x+\varepsilon+1}$. Moreover, for all sufficiently large $n$, the collocation-quadrature equations (3.4) possess a unique solution $u_{n}^{*}$ and, for $\varepsilon \leq \chi^{\prime} \leq \chi+\varepsilon$,

$$
\left\|u^{*}-u_{n}^{*}\right\|_{\alpha^{+}, \beta^{+}, \chi^{\prime}+1, q+1} \leq c \frac{\log ^{q}(n+1)}{n^{x+\varepsilon-\chi^{\prime}}}\left\|L_{n}\right\|_{\rho, \tau}\|f\|_{\rho, \tau, \chi+\varepsilon, q},
$$

where $c \neq c\left(n, \chi^{\prime}, \varepsilon\right)$.

Proof. As in the proof of Theorem 3.6 we get $(I+\widehat{V} K)^{-1} \in \mathscr{L}\left(\mathbf{C}_{\alpha^{+}, \beta^{+}}^{x^{\prime}+1, q+1}\right)$ for $0<\chi^{\prime} \leq \chi+\varepsilon$. By Proposition 2.6 and (2.7) we have, for $u \in \mathbf{C}_{\alpha^{+}, \beta^{+}}$,

$$
\begin{aligned}
\left\|\widehat{V} L_{n}\left(K_{n}-K\right) u\right\|_{\alpha^{+}, \beta^{+}, x^{\prime}+1, q+1} & \leq c\left\|L_{n}\left(K_{n}-K\right) u\right\|_{\gamma^{-}, \delta^{-}, \chi^{\prime}, q} \\
& \leq c n^{\chi^{\prime}}\left\|L_{n}\right\|_{\rho, \tau}\left\|\left(K_{n}-K\right) u\right\|_{\infty, \rho, \tau} .
\end{aligned}
$$

Consequently, in view of Lemma 3.8 and Corollary 3.5 ,

$$
\left\|\widehat{V}\left(L_{n} K_{n}-K\right) p\right\|_{\alpha^{+}, \beta^{+}, \chi^{\prime}+1, q+1} \leq c \frac{\log ^{q}(n+1)}{n^{x+\varepsilon-\chi^{\prime}}}\left\|L_{n}\right\|_{\rho, \tau}\|p\|_{\infty, \alpha^{+}, \beta^{+}}
$$

for all $p \in \Pi_{n}$. Choosing $\chi^{\prime}=\varepsilon$ and taking into account that $\Pi_{n}$ is invariant with respect to $I+\widehat{V} L_{n} K_{n}$, we obtain the invertibility of $I+\widehat{V} L_{n} K_{n}:\left(\Pi_{n},\|\cdot\|_{\alpha^{+}, \beta^{+}, \varepsilon+1, q+1}\right) \longrightarrow$ $\left(\Pi_{n},\|\cdot\|_{\alpha^{+}, \beta^{+}, \varepsilon+1, q+1}\right)$ for all sufficiently large $n$ as well as the uniform boundedness of the respective inverse operators. If $u_{n}^{*} \in \Pi_{n}$ is the solution of (3.4) and $P_{n} \in \Pi_{n}$ with $\left\|P_{n}-u^{*}\right\|_{\infty, \alpha^{+}, \beta^{+}}=E_{n}^{\alpha^{+}, \beta^{+}}\left(u^{*}\right)$, where $u^{*} \in \mathbf{C}_{\alpha^{+}, \beta^{+}}^{x+\varepsilon+1, q+1}$ is the solution of (3.1), then

$$
\begin{aligned}
\| P_{n} & -u_{n}^{*} \|_{\alpha^{+}, \beta^{+}, \varepsilon+1, q+1} \\
& \leq c\left\|\left(I+\widehat{V} L_{n} K_{n}\right) P_{n}-\widehat{V} L_{n} f\right\|_{\alpha^{+}, \beta^{+}, \varepsilon+1, q+1} \\
& =c\left\|\widehat{V}\left(f-L_{n} f\right)+(I+\widehat{V} K)\left(P_{n}-u^{*}\right)+\widehat{V}\left(L_{n} K_{n}-K\right) P_{n}\right\|_{\alpha^{+}, \beta^{+}, \varepsilon+1, q+1}
\end{aligned}
$$




$$
\begin{aligned}
\leq & c\left(\left\|\widehat{V}\left(f-L_{n} f\right)\right\|_{\alpha^{+}, \beta^{+}, \varepsilon+1, q+1}+\left\|P_{n}-u^{*}\right\|_{\alpha^{+}, \beta^{+}, \varepsilon+1, q+1}\right. \\
& \left.+\left\|\widehat{V}\left(L_{n} K_{n}-K\right) P_{n}\right\|_{\alpha^{+}, \beta^{+}, \varepsilon+1, q+1}\right) .
\end{aligned}
$$

Thus, with the help of Corollary 3.3, Corollary 3.2 and (3.15), we conclude

$$
\begin{aligned}
\| P_{n} & -u_{n}^{*} \|_{\alpha^{+}, \beta^{+}, \varepsilon+1, q+1} \\
& \leq c \frac{\log ^{q}(n+1)}{n^{X}}\left(\left\|L_{n}\right\|_{\rho, \tau}\|f\|_{\rho, \tau, \chi+\varepsilon, q}+\log (n+1)\left\|u^{*}\right\|_{\alpha^{+}, \beta^{+}, \chi+\varepsilon+1, q+1}\right),
\end{aligned}
$$

since $\left\|P_{n}\right\|_{\infty, \alpha^{+}, \beta^{+}} \leq 2\left\|u^{*}\right\|_{\infty, \alpha^{+}, \beta^{+}}$. It follows that

$$
\begin{aligned}
\| u^{*}- & u_{n}^{*} \|_{\alpha^{+}, \beta^{+}, x^{\prime}+1, q+1} \\
& \leq\left\|u^{*}-P_{n}\right\|_{\alpha^{+}, \beta^{+}, x^{\prime}+1, q+1}+n^{x^{\prime}-\varepsilon}\left\|P_{n}-u_{n}^{*}\right\|_{\alpha^{+}, \beta^{+}, \varepsilon+1, q+1} \\
& \leq c\left(\frac{\log ^{q+1}(n+1)}{n^{x+\varepsilon-\chi^{\prime}}}\left\|u^{*}\right\|_{\alpha^{+}, \beta^{+}, x+\varepsilon+1, q+1}+\frac{\log ^{q}(n+1)}{n^{x+\varepsilon-\chi^{\prime}}}\left\|L_{n}\right\|_{\rho, \tau}\|f\|_{\rho, \tau, x+\varepsilon, q}\right),
\end{aligned}
$$

and taking (3.12) into account, the theorem is proved.

If we combine the proofs of Theorems 3.7 and 3.9 we obtain the following theorem.

TheOREM 3.10. Assume $a=0, b=-1$ (that is, $\alpha=\beta=1 / 2$ ) and that

(a) $\Gamma \in \mathbf{C}_{0,0}^{\chi+\varepsilon, q}$ for some $\chi>0$ and $\varepsilon>0$,

(b) $h \in \mathbf{C}_{1 / 2,1 / 2, x}^{x+\varepsilon, q} \cap \mathbf{C}_{\nu, \zeta, t}^{x+\varepsilon, q}$ for some $\nu<1$ and $\zeta<1$,

(c) $\lim _{n \rightarrow \infty} \frac{\log ^{9}(n+1)}{n^{t^{\prime}}}\left\|L_{n}\right\|_{1 / 2,1 / 2}=0$ for some $0<\varepsilon^{\prime}<\min \{1, \chi\}$,

and that the homogeneous equation (3.1) possesses only the trivial solution in $\mathbf{C}_{1 / 2,1 / 2}^{\varepsilon+1, q+1}$. Then, for each $f \in \mathbf{C}_{1 / 2,1 / 2}^{x+\varepsilon, q}$, (3.1) has a unique solution $u^{*} \in \mathbf{C}_{1 / 2,1 / 2}^{x+\varepsilon+1, q+1}$. Moreover, for all sufficiently large $n,(3.4)$ has a unique solution $u_{n}^{*}$ and, for $\varepsilon \leq \chi^{\prime} \leq \chi+\varepsilon$,

$$
\left\|u^{*}-u_{n}^{*}\right\|_{1 / 2,1 / 2, \chi^{\prime}+1, q+1} \leq c \frac{\log ^{q}(n+1)}{n^{x+\varepsilon-\chi^{\prime}}}\left\|L_{n}\right\|_{1 / 2,1 / 2}\|f\|_{1 / 2,1 / 2, x+\varepsilon, q},
$$

where $c \neq c\left(n, \chi^{\prime}, f\right)$.

\section{Some remarks on a fast algorithm}

In [3, Section 4] the authors study a fast algorithm for (1.1) in the case where $a=0$ and $b=-1$, based on a collocation-quadrature method like (3.4), the computational complexity of which is $O(n \log n)$. Convergence properties of this algorithm are proved in weighted Sobolev norms, and the interested reader can also find numerical 
results for the collocation-quadrature method and the fast algorithm. The paper [7] investigates the uniform convergence of such a fast algorithm for Cauchy singular integral equations. Using the technique of [7] and the convergence results of the present paper it is possible to study the uniform convergence of the fast algorithm of [3]. Moreover, if we apply the following Lemma 4.1, which is an improvement of [7, Lemma 2.2] in the case where $\alpha=\beta=1 / 2$, then the considerations can be simplified in comparison to the proofs given in [7].

Let $S_{n}=S_{n}^{1 / 2,1 / 2}$ denote the Fourier projection operator with respect to the orthonormal system $\left\{p_{n}^{1 / 2,1 / 2}\right\}_{n=0}^{\infty}$, that is,

$$
S_{n} u=\sum_{j=0}^{n-1}\left\langle u, p_{n}^{1 / 2,1 / 2}\right\rangle_{1 / 2,1 / 2} p_{j}^{1 / 2,1 / 2}, \quad u \in \mathbf{L}_{1 / 2,1 / 2}^{2} .
$$

We remark that

$$
p_{n}^{1 / 2,1 / 2}(\cos \xi)=\frac{\sqrt{2} \sin (n+1) \xi}{\sin \xi}
$$

LEMMA 4.1. For all $f \in \mathbf{C}_{1 / 2,1 / 2}$ we have the estimate

$$
\left\|S_{n} f\right\|_{\infty, 1 / 2,1 / 2} \leq c \ln (n+1)\|f\|_{\infty, 1 / 2,1 / 2}, \quad n=1,2, \ldots,
$$

where $c \neq c(n, f)$.

ProOF. We use the relations ([12, Part I, Chapt. VII, Section 2])

$$
\cos \xi+\cos 2 \xi+\cdots+\cos n \xi=\frac{\sin ((2 n+1) \xi / 2)}{2 \sin (\xi / 2)}-\frac{1}{2}
$$

and

$$
\frac{1}{\pi} \int_{0}^{\pi / 2}\left|\frac{\sin (2 n+1) \xi}{\sin \xi}\right| d \xi<\frac{2+\ln n}{2}, \quad n=2,3, \ldots
$$

From (4.1) and (4.2) it follows that

$$
\begin{aligned}
& \sum_{j=0}^{n-1} p_{j}^{1 / 2,1 / 2}(\cos \tau) p_{j}^{1 / 2,1 / 2}(\cos \xi) \\
& \quad=\frac{2}{\sin \tau \sin \xi} \sum_{j=1}^{n} \sin j \tau \sin j \xi=\frac{1}{\sin \tau \sin \xi} \sum_{j=1}^{n}[\cos j(\tau-\xi)-\cos j(\tau+\xi)] \\
& \quad=\frac{1}{\sin \tau \sin \xi}\left[\frac{\sin \frac{2 n+1}{2}(\tau-\xi)}{2 \sin \frac{\tau-\xi}{2}}-\frac{\sin \frac{2 n+1}{2}(\tau+\xi)}{2 \sin \frac{\tau+\xi}{2}}\right]
\end{aligned}
$$


Since

$$
\left(S_{n} f\right)(x)=\frac{1}{\pi} \int_{-1}^{1} \sum_{j=0}^{n-1} p_{j}^{1 / 2,1 / 2}(t) p_{j}^{1 / 2,1 / 2}(x) f(t) v^{1 / 2,1 / 2}(t) d t,
$$

we conclude that

$$
\begin{aligned}
(\sin \xi) & \left(S_{n} f\right)(\cos \xi) \\
= & \frac{1}{2 \pi} \int_{0}^{\pi}\left[\frac{\sin \frac{2 n+1}{2}(\tau-\xi)}{\sin \frac{\tau-\xi}{2}}-\frac{\sin \frac{2 n+1}{2}(\tau+\xi)}{\sin \frac{\tau+\xi}{2}}\right] f(\cos \tau) \sin \tau d \tau \\
= & \frac{1}{2 \pi} \int_{-\pi}^{\pi} \frac{\sin \frac{2 n+1}{2}(\tau-\xi)}{\sin \frac{\tau-\xi}{2}} f(\cos \tau) \sin \tau d \tau \\
= & \frac{1}{2 \pi} \int_{-\pi}^{\pi} \frac{\sin \frac{2 n+1}{2} \theta}{\sin \frac{\theta}{2}} f(\cos (\xi+\theta)) \sin (\xi+\theta) d \theta \\
= & \frac{1}{2 \pi} \int_{0}^{\pi} \frac{\sin \frac{2 n+1}{2} \theta}{\sin \frac{\theta}{2}}[f(\cos (\xi+\theta)) \sin (\xi+\theta) \\
& +f(\cos (\xi-\theta)) \sin (\xi-\theta)] d \theta \\
= & \frac{1}{\pi} \int_{0}^{\frac{\pi}{2}} \frac{\sin (2 n+1) \delta}{\sin \delta}[f(\cos (\xi+2 \delta)) \sin (\xi+2 \delta) \\
& +f(\cos (\xi-2 \delta)) \sin (\xi-2 \delta)] d \delta .
\end{aligned}
$$

By (4.3) we can estimate $(x=\cos \xi)$

$$
\left|v^{1 / 2,1 / 2}(x)\left(S_{n} f\right)(x)\right|=\left|\sin \xi\left(S_{n} f\right)(\cos \xi)\right| \leq(2+\ln n)\left\|v^{1 / 2,1 / 2} f\right\|_{\infty},
$$

which proves the lemma.

\section{References}

[1] D. Berthold, W. Hoppe and B. Silbermann, "A fast algorithm for solving the generalized airfoil equation”, J. Comp. Appl. Math. 43 (1992) 185-219.

[2] M. R. Capobianco, "The stability and the convergence of a collocation method for a class of Cauchy singular integral equations", Math. Nachr. 162 (1993) 45-58.

[3] M. R. Capobianco, G. Criscuolo and P. Junghann, "A fast algorithm for Prandtl's integro-differential equation", J. Comp. Appl. Math. 77 (1997) 103-128.

[4] M. R. Capobianco, P. Junghanns, U. Luther and G. Mastroianni, "Weighted uniform convergence estimates of the quadrature method for Cauchy singular integral equations", in Singular Integral Operators and Related Topics (eds A. Böttcher and I. Gohberg), Operator theory Advances and Applications, Vol. 90, (Birkhäuser, 1996) 153-181. 
[5] M. R. Capobianco and M. G. Russo, "Uniform convergence estimates for a collocation method for the Cauchy singular integral equation", J. Integral Eqs Appl. 9 (1997) 1-25.

[6] P. Junghanns and U. Luther, "Cauchy singular integral equations in spaces of continuous functions and methods for their numerical solution", J. Comp. Appl. Math. 77 (1997) 201-237.

[7] P. Junghanns and $U$. Luther, "Uniform convergence of a fast algorithm for Cauchy singular integral equations", Linear Algebra Appl. 275-276 (1998) 327-347.

[8] P. Junghanns and U. Luther, "Uniform convergence of the quadrature method for Cauchy singular integral equations with weakly singular perturbation kernels", in Proc. of 3rd International Conference on Functional Analysis and Approximation Theory, (Acquafredda di Maratea, September, 1996) to appear.

[9] U. Luther, "Cauchy singular integral equations in weighted spaces of continuous functions", Preprint TU, Chemnitz-Zwickau, 1996.

[10] G. Mastroianni, "Uniform convergence of derivatives of Lagrange interpolation", J. Comp. Appl. Math. 43 (1992) 37-51.

[11] G. Mastroianni and M. G. Russo, "Lagrange interpolation in some weighted uniform spaces", in Proc. International Memorial Conference "D. S. Mitrinović", (Nis, Yugoslavia, June 1996).

[12] I. P. Natanson, Konstruktive Funktionentheorie (Akademie Verlag, Berlin, 1955).

[13] S. Prössdorf and B. Silbermann, Numerical Analysis for Integral and Related Operator Equations (Akademie Verlag, Berlin, 1991).

[14] G. Szegö, Orthogonal Polynomials (AMS, Providence, Rhode Island, 1939).

[15] P. Vértesi, "Weighted Lagrange interpolation on generalized Jacobi nodes", in Approximation Theory (eds N. K. Govil et al.), Monogr. Textbooks Pure Appl. Math. 212 (Marcel Dekker, New York, 1998) 489-497.

[16] P. Vértesi, "On the Lebesgue function of weighted Lagrange interpolation II", J. Austral. Math. Soc. (Ser. A) 65 (1998) 145-162. 